\section{REVISTA BRASILEIRA DE QUALIDADE DE VIDA}

\title{
Comparação entre autocuidado e estado menopausal em mulheres portadoras de Diabetes Mellitus tipo II
}

\section{Comparison between self-care and menopausal status in women with Diabetes Mellitus type II}

\begin{abstract}
Ronilson Ferreira Freitas Faculdades Integradas Pitágoras de Montes Claros - FIPMoc - Montes Claros - Minas Gerais - Brasil Universidade Cândido Mendes - UCAM - Rio de Janeiro - Rio de Janeiro - Brasil ronnypharmacia@gmail.com

Débora Ribeiro Vieira Faculdades Integradas Pitágoras de Montes Claros - FIPMoc - Montes Claros - Minas Gerais - Brasil debora_ribeiro_vieira@hotmail.com

Tahiana Ferreira Freitas Faculdade de Saúde Ibituruna - FASI - Montes Claros - Minas Gerais - Brasil tahiana.fono@gmail.com

Vivianne Margareth Chaves Pereira Reis Faculdades Integradas Pitágoras de Montes Claros - FIPMoc - Montes Claros - Minas Gerais - Brasil Faculdades Unidas do Norte de Minas - FUNORTE - Montes Claros - Minas Gerais - Brasil viola.chaves@yahoo.com.br

Betânia Maria Araújo Passos Universidade Estadual de Montes Claros - UNIMONTES - Montes Claros - Minas Gerais - Brasil betahidro@yahoo.com.br

Josiane Santos Brant Rocha Universidade Estadual de Montes Claros - UNIMONTES - Montes Claros - Minas Gerais - Brasil Faculdades Integradas Pitágoras de Montes Claros - FIPMoc - Montes Claros - Minas Gerais - Brasil josianenat@yahoo.com.br
\end{abstract}

\section{RESUMO}

OBJETIV0: Comparar as variáveis de autocuidado com o estado menopausal de mulheres climatéricas portadoras de Diabetes Mellitus Tipo II.

MÉTODOS: Estudo transversal e quantitativo, composto por mulheres climatéricas portadoras de Diabetes Mellitus Tipo II, com idade entre 40 e 65 anos, residentes na área urbana de Montes ClarosMG atendidas pelas Unidades Básicas de Saúde. A amostra foi composta por 96 mulheres. Os instrumentos utilizados para a coleta de dados foram questionários sociodemográfico, clínico e de autocuidado e avaliação antropométrica. Para caracterizar o universo amostral pesquisado foi utilizada a análise descritiva com média e desvio padrão. Para averiguar a existência de associação entre as variáveis categóricas foi utilizado o teste Chi-Square. Em seguida, foi verificada a alocação de associação entre categorias através dos resíduos ajustados, e considerado como nível de significância o valor de $\mathrm{p}<0,05$. 
RESULTADOS: Houve prevalência de mulheres com faixa etária entre 59 a 65 anos (56,3\%), da raça parda $(49,0 \%)$, com menos de 5 anos completos de estudo (47,9\%), que não exercem atividade remunerada $(77,1 \%)$, com renda familiar de 1 a 2 salários mínimos $(53,1 \%)$ e com companheiro fixo (65,6\%). Quanto à prática de atividade física e tabagismo, 54,2\% das mulheres não praticam e 83,3\% não são fumantes. Quando analisado o perfil ginecológico, houve um maior número de mulheres pósmenopausadas $(68,8 \%)$, cuja menopausa ocorreu de forma natural $(83,3 \%)$, o sintoma prevalente da menopausa foram os fogachos, a maioria das mulheres responderam ter tido três ou mais partos $(67,7 \%)$ e 50,0\% responderam praticar relações sexuais. Na comparação do autocuidado com estado menopausal, houve efeito significante no item avaliou 'açúcar no sangue', 'uso de medicamentos regularmente', 'toma número indicado de comprimidos de diabetes' e 'examinou os pés', destacando a maior adesão por parte das mulheres pós-menopáusicas.

CONCLUSÕES: As mulheres pós-menopausadas possuem nível de autocuidado maiores quando comparado com as mulheres na pré e na perimenopausa.

PALAVRAS-CHAVE: Diabetes Mellitus. Estado Menopausal. Autocuidado.

\section{ABSTRACT}

OBJECTIVE: Compare the variables of self-care with the menopausal status of women with perimenopausal women with Diabetes Mellitus type II.

METHODS: Transversal and quantitative study, consisting of menopausal women suffering from Diabetes Mellitus Type II, aged between 40 and 65 years living in the urban area Claros-MG Montes served by Basic Health Units The sample consisted of 96 women. The instruments used for data collection were sociodemographic, clinical and anthropometric assessment questionnaires and selfcare. To characterize the sample universe researched the descriptive analysis with mean and standard deviation was used. To investigate the possible association between categorical variables, the ChiSquare test was used. Next, we investigated the association between categories of allocation through the adjusted residuals, and found the significance level of $\mathrm{p}$ value $<0.05$.

RESULTS: The prevalence of women aged between 59-65 years $(56,3 \%)$, mulatto $(49,0 \%)$, with less than 5 years of schooling $(47,9 \%)$, not pursuing gainful activity $(77,1 \%)$, with family income 1-2 minimum wages $(53,1 \%)$ and with a steady partner $(65,6 \%)$. Regarding physical activity and smoking, $54,2 \%$ of women and $83,3 \%$ do not practice are non-smoking. When analyzed gynecological profile, there was a higher number of postmenopausal women $(68,8 \%)$, whose menopause occurred naturally $(83,3 \%)$, the most prevalent symptom of menopause are hot flashes, most women reported having had three or more births $(67,7 \%)$ and 50,0\% reported practicing sex. Compared with self-care, menopausal status, a significant effect on the evaluated item 'blood sugar', 'use of medicines regularly', 'takes indicated number of diabetes pills' and 'examined the feet', highlighting the increased ownership by the postmenopausal women.

CONCLUSIONS: Those postmenopausal women have a higher level of self-care when compared with women in pre-and perimenopausal.

KEYWORDS: Diabetes Mellitus. Menopausal status. Self-Care.

\section{Introdução}

O climatério é uma fase da vida caracterizada pela transição do período reprodutivo para o não-reprodutivo, iniciado em torno dos 40 anos e terminado em torno dos 65 anos (POLISSENI et al., 2009). Começa pela passagem do ciclo ovulatório potencialmente fértil para um período de falência ovariana, caracterizado pelo hipoestrogenismo, tem como consequência o fim do ciclo 
menstrual, período conhecido como menopausa, e o surgimento dos sintomas característicos (PARANDAVAR et al., 2014; CABRAL et al., 2012).

No período do climatério as mulheres apresentam uma maior tendência ao ganho de peso. A tendência gera uma grande preocupação, já que mulheres obesas, principalmente na pós-menopausa, apresentam uma maior tendência à hipertensão, Diabetes Mellitus tipo II (DM II), câncerdemama, doendométrioenocólon (PARK et al., 2014; LORENZI et al., 2005).

O DM II é um dos mais graves problemas de saúde pública em todo o mundo, particularmente no Brasil, pela alta prevalência e por se destacar como importante fator de risco cardiovascular. Constitui-se numa síndrome do metabolismo defeituoso de carboidratos, lipídios e proteínas, causada tanto pela ausência de secreção de insulina, quanto pela diminuição da sensibilidade dos tecidos à insulina, o que se traduz laboratorialmente pela elevação da taxa de glicemia (SILVA; SIMÕES; LEITE, 2007).

Nos últimos anos, devido à crescente urbanização e consequente alteração no estilo de vida, o número de pessoas portadoras do DM II tem aumentado significativamente (GROSS et al., 2002). O DM II é mais frequente após os 40 anos de idade, com o maior pico em torno dos 60 anos (TEED; LOMBARD; DEEKS, 2010). Diante deste aspecto, é possível inferir que uma das classes que se predispõe a ser acometida pelo DM II são mulheres climatéricas, em decorrência da faixa etária (LORENZI et al., 2005).

Nesta perspectiva, o objetivo do presente estudo foi comparar as variáveis de autocuidado com o estado menopausal de mulheres climatéricas portadoras de DM II.

\section{Metodologia}

Foi realizado um estudo transversal, descritivo, de caráter quantitativo, com amostra composta por 96 mulheres climatéricas, portadoras de DM II, atendidas em Estratégias de Saúde da Família (ESF) conveniadas com as Faculdades Integradas Pitágoras de Montes Claros, entre março e junho de 2012.

Os critérios de inclusão adotados foram gênero do sexo feminino, idade entre 40 e 65 anos, frequentar as ESFs onde foram coletados os dados e aceitarem participar da pesquisa, assinando o Termo de Consentimento Livre e Esclarecido.

A pesquisa abrangeu a avaliação sociodemográfica, ginecológica e do autocuidado. Foi utilizado como instrumento de coleta de dados questionários semiestruturados.

Dentre os dados coletados na avaliação sociodemográfica e clínica estavam a idade, a raça, os anos de estudo completo, a atividade remunerada, a renda em salários mínimos, o estado marital, a prática de atividade física e o tabagismo. Para a avaliação dos fatores ginecológicos foi questionado o estado menopausal, o tipo de menopausa, os sintomas da menopausa, o número de partos e de relações sexuais, e para a variável comportamento de autocuidado foram aplicadas questões sobre a alimentação geral e específica, a atividade física, a monitoração da glicemia e o uso de medicamentos.

Para a análise da aderência aos itens do questionário de autocuidado foram parametrizados em dias por semana, de zero a sete, sendo zero a situação menos desejável e sete a mais favorável. Nos itens da dimensão alimentação específica, relativa ao consumo de alimentos ricos em gordura e doces, os valores foram invertidos (se $7=0,6=1,5=2,4=3,3=4,2=5,1=6,0=7$ ), como sugeridos no Summary of Diabetes Self-Care Activities Questionnaire (SDSCA).

Para caracterizar o universo amostral pesquisado foi utilizada a análise descritiva com média e desvio padrão. Para averiguar a existência de associação entre as variáveis categóricas foi utilizado o teste Chi-Square. Em seguida, foi verificada a alocação de associação entre categorias através dos resíduos ajustados, e considerado como nível de significância o valor de $\mathrm{p}<0,05$, estabelecido pelo pacote Statistical Package For The Social Science (SPSS), versão 14.0.

O Projeto de Pesquisa foi submetido para apreciação do Comitê de Ética em Pesquisa com seres humanos, das Faculdades Integradas Pitágoras de Montes Claros, e aprovado sob parecer $038757 / 2012$. 


\section{Resultados}

A distribuição das participantes com relação às variáveis sociodemográficas (idade, raça, instrução, atividade remunerada, renda familiar e estado marital) e clínicas (prática de atividade física, doenças, tabagismo e hipertensão) encontra-se na Tabela 1:

Tabela 1 - Perfil sociodemográfico da população analisada

\begin{tabular}{|c|c|c|}
\hline $\begin{array}{l}\text { Fatores Sociodemográficos e } \\
\text { Clínicos }\end{array}$ & Variáveis & $(\%)(\mathbf{n})$ \\
\hline \multirow{5}{*}{ Idade } & 40 a 44 anos & $6,3-6$ \\
\hline & 45 a 49 anos & $16,7-16$ \\
\hline & 50 a 54 anos & $15,6-15$ \\
\hline & 55 a 59 anos & $5,2-5$ \\
\hline & 59 a 65 anos & $56,2-54$ \\
\hline \multirow{5}{*}{ Raça } & Branca & $32,3-31$ \\
\hline & Negra & $16,7-16$ \\
\hline & Parda & $49,0-47$ \\
\hline & Amarela & $1,0-1$ \\
\hline & Índia & $1,0-1$ \\
\hline \multirow{3}{*}{ Anos de estudo completo } & Menos de 5 anos & $47,9-46$ \\
\hline & Entre 5 e 9 anos & $32,3-31$ \\
\hline & 10 anos ou mais & $19,8-19$ \\
\hline \multirow[t]{2}{*}{ Atividade remunerada } & $\operatorname{Sim}$ & $22,9-22$ \\
\hline & Não & $77,1-74$ \\
\hline \multirow{3}{*}{ Renda em salários mínimos } & Menos de 1 & $32,3-31$ \\
\hline & De 1 a 2 & $53,1-51$ \\
\hline & 3 ou mais & $14,6-14$ \\
\hline \multirow[t]{4}{*}{ Estado marital } & Companheiro fixo & $65,6-63$ \\
\hline & Sem companheiro fixo & $34,4-33$ \\
\hline & Não pratica & $54,2-52$ \\
\hline & $\begin{array}{l}\text { Menos que três vezes por semana (em } \\
\text { menos que } 60 \text { minutos }\end{array}$ & $25,0-24$ \\
\hline \multirow[t]{2}{*}{ Prática de atividade física } & $\begin{array}{l}\text { Três vezes por semana (por } 60 \\
\text { minutos ou mais) }\end{array}$ & $8,3-8$ \\
\hline & $\begin{array}{l}\text { Mais que três vezes por semana (por } \\
60 \text { min ou mais) }\end{array}$ & $12,5-12$ \\
\hline \multirow[t]{2}{*}{ Tabagismo } & $\operatorname{Sim}$ & $16,7-16$ \\
\hline & Não & $83,3-80$ \\
\hline
\end{tabular}

Nota: (\%) - Porcentagem observada; (n) - Tamanho da Amostra. Fonte: Autoria própria (2013).

A análise descritiva das participantes com relação aos fatores ginecológicos (estado menopausal, o tipo de menopausa, os sintomas da menopausa, o número de partos e a prática de relações sexuais) encontra-se na Tabela 2: 
Tabela 2 - Fatores ginecológicos da população analisada

\begin{tabular}{|c|c|c|}
\hline Fatores Ginecológicos & Variáveis & $(\%)(\mathrm{n})$ \\
\hline Estado menopausal & $\begin{array}{l}\text { Ciclo menstrual regular } \\
\text { Pré-menopausa } \\
\text { Perimenopausa } \\
\text { Pós-menopausa }\end{array}$ & $\begin{array}{c}10,4-10 \\
9,4-9 \\
11,5-11 \\
68,7-66\end{array}$ \\
\hline Tipo de menopausa & $\begin{array}{l}\text { Natural } \\
\text { Induzida } \\
\text { Não está }\end{array}$ & $\begin{array}{c}83,3-80 \\
7,3-7 \\
9,4-9\end{array}$ \\
\hline Sintomas da menopausa & $\begin{array}{l}\text { Fogaços } \\
\text { Diminuição do desejo sexual } \\
\text { Outros } \\
\text { Sensibilidade emocional } \\
\text { Ausência de sintomas }\end{array}$ & $\begin{array}{c}46,9-45 \\
6,3-6 \\
14,6-14 \\
18,7-18 \\
13,5-13\end{array}$ \\
\hline Número de partos & $\begin{array}{l}\text { Nenhum } \\
\text { Um ou dois } \\
\text { Três ou mais }\end{array}$ & $\begin{array}{c}7,3-7 \\
25,0-24 \\
67,7-65\end{array}$ \\
\hline Relações sexuais & $\begin{array}{l}\text { Sim } \\
\text { Não } \\
\text { Não respondeu }\end{array}$ & $\begin{array}{c}50,0-48 \\
43,7-42 \\
6,3-6\end{array}$ \\
\hline
\end{tabular}

A comparação entre autocuidado e estado menopausal, encontra-se disposta na Tabela 3:

Tabela 3 - Comparação entre autocuidado e estado menopausal na população analisada

\begin{tabular}{|c|c|c|c|c|c|c|}
\hline \multirow{2}{*}{ Autocuidado } & \multicolumn{4}{|c|}{ Estado Menopausal } & \multirow{2}{*}{$\mathrm{F}$} & \multirow{2}{*}{$\mathrm{p}^{*}$} \\
\hline & CMR & Pré & Peri & Pós & & \\
\hline Dieta saudável & 4,90 & 4,78 & 5,09 & 5,29 & 0,210 & 0,889 \\
\hline Seguiu orientações alimentares de profissionais & 5,40 & 3,78 & 3,91 & 4,23 & 0,771 & 0,513 \\
\hline Comeu no mínimo cinco frutas e/ou vegetais & 4,40 & 4,78 & 4,27 & 5,03 & 0,441 & 0,724 \\
\hline Alimentos ricos em gordura & 2,80 & 2,56 & 3,18 & 3,67 & 0,98 & 0,406 \\
\hline Ingesta de doces & 0,50 & 1,22 & 1,18 & 1,21 & 0,571 & 0,636 \\
\hline Praticou atividade física excluindo atividades cotidianas & 1,70 & 0,89 & 1,64 & 1,76 & 0,380 & 0,767 \\
\hline Praticou atividade física por no mínimo 30 minutos & 3,10 & 1,67 & 1,73 & 1,85 & 0,826 & 0,483 \\
\hline Avaliou açúcar no sangue & 3,00 & 2,33 & 0,73 & 1,89 & $2,722 *$ & $0,049 *$ \\
\hline Avaliou açúcar no sangue as vezes recomendada & 0,50 & 2,33 & 0,55 & 1,65 & 2,091 & 0,107 \\
\hline Usa medicamentos regularmente & 6,40 & 5,67 & 4,36 & 6,08 & $2,940 *$ & $0,037 *$ \\
\hline Toma insulina regularmente & 3,50 & 3,44 & 2,45 & 3,14 & 0,256 & 0,857 \\
\hline Toma número indicado de comprimidos de diabetes & 5,50 & 4,33 & 3,36 & 4,15 & $0,913^{*}$ & $0,429 *$ \\
\hline Examinou os calçados antes de usar & 3,50 & 3,44 & 2,45 & 3,14 & 0,256 & 0,857 \\
\hline Secou espaços entre dedos dos pés depois de lavá-los & 5,50 & 4,33 & 3,36 & 4,15 & 0,931 & 0,429 \\
\hline Examinou os seus pés & 1,00 & 3,78 & 4,82 & 3,61 & $3,259 *$ & $0,011^{*}$ \\
\hline
\end{tabular}

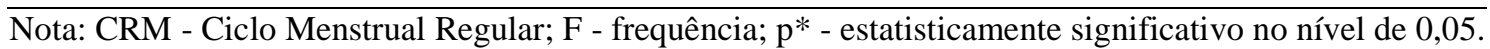
Fonte: Autoria própria (2013). 


\section{Discussão}

Em relação aos resultados obtidos, ao comparar o autocuidado e o estado menopausal, houve significância para os quesitos: em quantos dos últimos sete dias 'avaliou o açúcar no sangue'; em quantos dos últimos sete dias 'examinou os pés'; em quantos dos últimos sete dias 'usou medicamentos regularmente'; em quantos dos últimos sete dias 'tomou o número indicado de comprimidos do diabetes'.

Quando avaliado o quesito em quantos dos últimos sete dias 'avaliou o açúcar no sangue' foi possível observar uma baixa aderência por parte das mulheres na perimenopausa $(0,73)$ e na pósmenopausa (1,89). Segundo Ferraz et al. (2013), o diabetes é uma doença assintomática, de possíveis instalações de comorbidades em órgãos-alvo sem a percepção do portador. Com efeito, torna-se necessária a manutenção de níveis glicêmicos adequados. A baixa adesão ao monitoramento é um fator preocupante e agravante do risco de desenvolvimento de complicações do estado clínico da paciente, principalmente no período que antecede a pós-menopausa, onde os riscos aumentam. Dentre os riscos que corre um paciente que não faz o controle adequado do diabetes pode-se citar a disfunção e a falência de órgãos, como rins, olhos, nervos, coração e vasos sanguíneos, acarretando a neuropatia, a nefropatia, a retinopatia, o infarto do miocárdio, acidentes vasculares e infecções (RAMOS; FERREIRA, 2011).

Coelho e Silva (2006) ressaltam a importância da atenção aos pés e o uso de calçados confortáveis, já que há no país um grande número de internações de pessoas diabéticas devido a complicações nos pés. Essas complicações, precedidas por lesões, podem evoluir para amputações, normalmente ocasionadas por trauma extrínseco, principalmente com o uso de calçados inadequados. Entretanto, quando avaliado o item em quantos dos últimos sete dias 'examinou os pés', a maior aderência ao cuidado com os pés foi para as mulheres na perimenopausa $(4,82)$, o que vai de acordo com os resultados encontrados por Ochoa-Vigo et al. (2006).

No que se refere ao uso da medicamentos nos últimos sete dias, a amostra apresenta resultados desejáveis para o uso correto dos medicamentos para diabetes, dados que vão de acordo aos obtidos nos estudos de Assunção e Ursine (2008), justificando que com o envelhecimento populacional e a aposentadoria, essas mulheres têm maior tempo livre e maior disponibilidade para cuidar de sua saúde e procurar os serviços de saúde, facilitando assim o acesso aos serviços médicos e à maiores informações das melhores condutas para o tratamento.

Apesar das recomendações clínicas e a difusão das melhores formas de tratamento e controle do diabetes, a manutenção contínua do DM II é tida como o principal problema para suas portadoras. Apesar das mudanças sociais ocorridas no Brasil, muitas mulheres que não trabalham fora e ficam em casa apresentam significante estresse interpessoal familiar, tomando para elas as responsabilidades do cotidiano, nos cuidados dos membros da família e na mediação de conflitos familiares. $\mathrm{O}$ estresse e tantas responsabilidades fazem com que muitas mulheres não consigam controlar adequadamente os níveis de açúcar no sangue e ficam expostas aos riscos de complicações da doença (SILVA; HEGADOREN; LASIUK, 2012).

\section{Conclusão}

A capacidade de autocuidado das mulheres portadoras de DM II apresentaram médias que estão acima do preconizado para um individuo saudável. Foi observado que as mulheres pósmenopausadas possuem nível de autocuidado maiores quando comparado com as mulheres na pré e na perimenopausa. É imprescindível que sejam estudados novos métodos e ações para o incentivo à prática do autocuidado buscando a melhoria do comportamento das mulheres frente a essa patologia. 


\section{Referências}

ASSUNÇÃO, T. S.; URSINE, P. G. S. Estudo de fatores associados à adesão ao tratamento não farmacológico em portadores de diabetes mellitus assistidos pelo Programa Saúde da Família, Ventosa, Belo Horizonte. Revista Ciência \& Saúde Coletiva, v. 13, sup. 2, p. 2189-2197, 2008.

CABRAL, P. U. L.; CANÁRIO, A. C. G.; SPYRIDES, M. H. C.; UCHÔA, S. A. C.; ELEUTÉRIO JÚNIOR, J.; AMARAL, R. L. G.; GONÇALVES, A. K. S. Influência dos sintomas climatéricos sobre a função sexual de mulheres de meia-idade. RevistaBrasileira de Ginecologia e Obstetrícia, v. 34, n. 7, 329-334, 2012. crossef

COELHO, M. S.; SILVA, D. M. G. V. da. Grupo educação-apoio: visualizando o autocuidado com os pés de pessoas com diabetes mellitus. Revista Ciência, Cuidado e Saúde. Maringá, v. 5, n. 1, p. 11-15, jan./abr. 2006.

FERRAZ, R. R. N.; SILVA, M. C. M. da; SILVA, S. C.; NIGRO, C. A.; FORNARI, J. V.; BARNABÉ, A. S. Avaliação do conhecimento dos portadores de Diabetes Melittus sobre a importância da manutenção dos níveis glicêmicos para prevenção da nefropatia diabética. Revista Saúde e Biologia, v. 8, n. 3, p. 49-55, ago./dez. 2013.

GROSS, J. L.; SILVEIRO, S. P.; CAMARGO, J. L.; REICHELT, A. J.; AZEVEDO, M. J.de. Diabetes Melito: diagnóstico, classificação e avaliação do controle glicêmico. Arquivos Brasileiros de Endocrinologia \& Metabolismo, v. 46, n. 1, p. 11-26, 2002. crossef

LORENZI, D. R. S. de; BASSO, E.; FAGUNDES, P. de O.; SACILOTO, B. Prevalência de sobrepeso e obesidade no climatério. Revista Brasileira de Ginecologia e Obstetrícia, Rio de Janeiro, v. 27, n. 8, p. 479-484, 2005. Crossef

OCHOA-VIGO, K.; TORQUATO, M. T. C. G.; SILVÉRIO, I. A. S.; QUEIROZ, F. A.; DE-LATORRE-UGARTE-GUANILO, M. C.; PACE, A. E. Caracterização de pessoas com diabetes em unidades de atenção primária e secundária em relação a fatores desencadeantes do pé diabético. Acta Paulista de Enfermagem, v. 19, n. 3, p. 296-303, 2006. Crossef

PARANDAVAR, N.; MOSALANEJAD, L.; RAMEZANLI, S.; GHAVI, F. Menopause and Crisis? Fake or real: comprehensive search to The Depth of Crisis Experienced: a mixed-method study.Global Journal of Health Science, v. 6, n. 2, p. 246-255, 2014. Crossef

PARK, J. Y.; CHO, J. H.; MIN, J. Y.; KIM, D. Y.; KIM, J. H.; KIM, Y. M.; KIM, Y. T.; NAM, J. H. Impact of body mass index on the prognosis of korean women with endometrioid adenocarcinoma of the uterus: a cohort study. Archive of Obstetrics and Gynecology Science, v. 57, n. 2, p. 115-120, 2014. crossef

POLISSENI, Á. F.; ARAÚJO, D. A. C.; POLISSENI, F.; MOURÃO JUNIOR, C. A.; POLISSENI, J.; FERNANDES, E. S.; GUERRA, M. O. Depressão e ansiedade em mulheres climatéricas: fatores associados. Revista Brasileira de Ginecologia e Obstetrícia, v. 31, n. 1, p. 28-34, 2009. Crossef

RAMOS, L.; FERREIRA, E. A. P. Fatores emocionais, qualidade de vida e adesão ao tratamento em adultos com diabetes tipo 2. Revista Brasileira de Crescimento e Desenvolvimento Humano, v. 21, n. 3, p. 867-877, 2011. 
SILVA, D. M. G. V. da; HEGADOREN, K.; LASIUK, G. As perspectivas de donas de casa brasileiras sobre a sua experiência com diabetes mellitus tipo 2. Revista Latino-Americana de Enfermagem, v. 20, n.3, maio-jun. 2012.

SILVA, R. C. P.; SIMÕES, M. J. S.; LEITE, A. A. Fatores de risco para doenças cardiovasculares em idosos som diabetes mellitus tipo 2. Revista Ciências Farmacêuticas Básica e Aplicada, v. 28, n. 1, p. 113-121, 2007.

TEED, H. J.; LOMBARD, C.; DEEKS, A. A. Obesity, metabolic complications and the menopause: an opportunity for prevention. Climateric, v. 13, n. 3, p. 203-209, 2010. Crossef 